\title{
Contribuições da análise espacial para a compreensão da dinâmica de transmissão da dengue: revisão integrativa
}

\section{Contributions of spatial analysis to the comprehension of dynamics of dengue transmission: integrative review}

\author{
Lacita Menezes Skalinski1 ${ }^{1,2}$ (D), Maria da Conceição Nascimento Costa $^{3}$ (D) , Maria da Glória Lima Teixeira ${ }^{3}$ (D)
}

1. Docente do curso de Enfermagem da Universidade Estadual de Santa Cruz (UESC), Ilheus, BA, Brasil. 2. Doutoranda em Saúde Coletiva pela Universidade Federal da Bahia (UFBA), Salvador, BA, Brasil. 3. Docente do Instituto de Saúde Coletiva da Universidade Federal da Bahia (UFBA), Salvador, BA, Brasil

\section{Resumo}

Introdução: a análise espacial tem sido utilizada pela vigilância epidemiológica como estratégia para identificação de espaços urbanos sob maior risco de doenças. Nesse contexto, as ferramentas da informática em saúde podem auxiliar para a compreensão dos fenômenos envolvidos na difusão da dengue nos espaços de aglomeração populacional, elucidando questões relativas aos movimentos espaciais de sua ocorrência e subsidiando o delineamento de programas de combate a seu principal vetor. Objetivo: sintetizar e discutir as informações mais relevantes produzidas pelos estudos de análise espacial de dengue, as quais vêm contribuindo para ampliar o entendimento de sua dinâmica de transmissão, no propósito da aplicação na prática da vigilância epidemiológica. Método: trata-se de uma pesquisa de revisão integrativa de literatura, realizada com publicações de 1945 a 2017, na Web of Science sobre dengue, com uso dos descritores e operadores "Spatial Analysis" AND "Dengue" AND "Urban Area". Resultados: foram selecionados 35 artigos. 0 perfil das produções permitiu a identificação de sete assuntos mais abordados: mobilidade urbana, densidade populacional, fatores sociodemográficos, disponibilidade de água, temperatura, vegetação e urbanização. Considerações finais: a análise espacial, por meio dos Sistemas de Informação Geográfica, é uma ferramenta muito útil para o estudo das dinâmicas de transmissão da dengue, pois possibilita o conhecimento de áreas, períodos e fatores sociais e ambientais de maior risco, a fim de produzir alertas para os serviços de saúde.

Palavras-chave: Análise espacial. Dengue. Área urbana.

\begin{abstract}
Introduction: The spatial analysis has been used by epidemiological surveillance as a strategy to identify urban spaces at greater risk of diseases. In this context, the tools of computer sciences apllied to health can help to understand the phenomena involved in the diffusion of dengue in areas of high population density. It clarifies the issues related to the spatial movements of dengue occurrence and subsidize the design of programs to combat its main vector. Objective: this study aimed to synthesize and discuss the most relevant information produced by the studies about spatial analysis of dengue, which have contributed to enlarge the understanding of its transmission dynamics, in the purpose of the practical application of epidemiological surveillance. Method: this is an integrative literature review carried out with publications from 1945 to 2017 in the Web of Science. The descriptors and boolean operators used were "Spatial Analysis" AND "Dengue" AND “Urban Area”. Results: 35 papers were selected. The analysis of the productions allowed the identification of seven subjects: urban mobility, population density, sociodemographic factors, availability of water, temperature, vegetation and urbanization. Final considerations: the spatial analysis, through the Geographic Information Systems, is a very useful tool to the study of dengue transmission dynamics, because it allows the knowledge of areas, periods and social and environmental factors of greater risk, in order to produce alerts for health services.
\end{abstract}

Key words: Spatial Analysis; Dengue; Urban Area.

\section{INTRODUÇÃO}

Na Saúde Pública, o conceito de espaço já era usado há mais de 2.000 anos e tem sido reformulado, moldando-se de acordo com a concepção de saúde-doença vigente em cada época. De Hipócrates (séc. V a.C.) a Milton Santos (1996), o espaço deixou de ser considerado apenas como uma delimitação geográfica, passando a contemplar as relações e as dinâmicas sociais, econômicas e políticas que o modificaram no decorrer da História ${ }^{1}$. Nesse sentido, o desenvolvimento da análise espacial representou uma valiosa ferramenta para a compreensão sobre como determinado contexto afeta a saúde da população e os grupos populacionais.

A evolução da concepção de espaço, em Epidemiologia, passou a ser orientada a partir de seu entendimento como o lugar onde os agentes infecciosos circulam e, desse modo, possibilitou os avanços teóricos alcançados pela Geografia². Na pesquisa epidemiológica, a estratégia de análise espacial foi incorporada na perspectiva de melhor conhecer e entender o padrão de distribuição das doenças e agravos à saúde da população, assim como seus determinantes em espaços delimitados. No âmbito da vigilância das doenças transmissíveis, o espaço, enquanto categoria de análise, foi incorporado às suas práticas por permitir identificar áreas de aglomeração de eventos de saúde, ou seja, áreas de maior risco de ocorrência de doenças, informação que representa importante subsídio para o planejamento das ações de prevenção e controle ${ }^{3,4}$, bem como para avaliação e monitoramento de aspectos ambientais, condicionantes geográficos e socioeconômicos relacionados à

Correspondência: Lacita Menezes Skalinski. Instituto de Saúde Coletiva da Universidade Federal da Bahia (UFBA). Rua Basílio da Gama, s/n. Campus Universitário Canela , Canela, Salvador - CEP: 40.110-040. E-mail: Imskalinski@yahoo.com.br Conflito de interesse: Não há conflito de interesse por parte de qualquer um dos autores. Recebido em: 3 Maio 2018; Revisado em: 28 Maio 2018; 6 Nov 2018 Aceito em: 8 Nov 2018 
doença de interesse.

As técnicas de geoprocessamento e a geoestatística vêm possibilitando que os estudos ecológicos, de certa forma, tornem mais evidentes a participação da conjunção de fatores, inclusive os contextuais, na determinação da doença por incorporar efeitos das características específicas de cada espaço social. Embora com limitações, essa abordagem contribui para que a epidemiologia possa integrar, dialeticamente, o social com o natural, conforme sustentado pelos pensadores da epidemiologia social ${ }^{5}$.

No Brasil, essas estratégias metodológicas vêm sendo aplicadas de modo mais rotineiro desde os anos de $1980^{6}$, principalmente no campo da vigilância epidemiológica, na medida em que os espaços urbanos passaram a se destacar devido à rápida disseminação de doenças transmissíveis. Isso se deveu à formação de grandes adensamentos populacionais, desencadeada pelo processo de intensa migração do campo para as cidades, a partir da segunda metade do século $X X$, os quais configuram condições fundamentais para a difusão de agentes infecciosos, especialmente aqueles cuja cadeia epidemiológica envolve a participação de reservatórios ou vetores urbanos ${ }^{7}$.

Um dos exemplos mais emblemáticos desse processo foi a reemergência do vírus da dengue no Rio de Janeiro, em 1986, quando produziu mais de 47 mil casos, valor superior ao de cada uma das demais doenças de notificação compulsória. Enquanto o Brasil foi eliminando e/ou controlando a morbimortalidade pela maioria das doenças infecciosas por meio de intervenções de Saúde Pública, a dengue passou a se constituir, nas últimas três décadas, em um dos principais problemas de saúde desse campo, na medida em que vem produzindo epidemias de grande magnitude, em mais de $90 \%$ das cidades do país, sem que se vislumbre perspectiva de controle e /ou eliminação do seu principal transmissor ${ }^{8}$.

Nesse contexto, é da maior relevância lançar mão das ferramentas da informática em saúde para melhor entender os fenômenos envolvidos na difusão das arboviroses nos espaços de aglomeração populacional, tomando como exemplo a dengue. Essas informações contribuem para a elucidação de questões relativas aos movimentos espaciais de sua ocorrência assim como para subsidiar o delineamento de programas de combate a seu principal vetor. O objetivo deste estudo foi sintetizar e discutir as informações mais relevantes produzidas pelos estudos de análise espacial de dengue, as quais vêm contribuindo para ampliar o entendimento da sua dinâmica de transmissão no propósito da aplicação na prática da vigilância epidemiológica.

\section{MÉTODOS}

Este estudo foi realizado em maio de 2018, mediante uma revisão integrativa, método de pesquisa bastante utilizado no âmbito da Prática Baseada em Evidências, que se caracteriza por propiciar uma síntese do conhecimento científico sobre o tema investigado, voltada em especial para sua aplicabilidade na prática da assistência à saúde na medida em apresenta resultados que dão suporte para a tomada de decisão e aprimoramento na prática clínica. Esse método de revisão é constituído de seis etapas: 1 ) identificação do tema e da questão norteadora; 2) estabelecimento dos critérios de inclusão e exclusão; 3) coleta de dados dos artigos selecionados; 4) análise crítica dos artigos visando classificar as evidências encontradas; 5) interpretação dos resultados; 6) síntese do conhecimento ${ }^{9-11}$.

A base de dados utilizada na busca foi a Web of Science e os descritores (MeSH terms) utilizados foram: Spatial Analysis, Dengue e Urban Area. Com apoio dos operadores booleanos, foi realizada a busca por meio da seguinte combinação: Spatial Analysis AND Dengue AND Urban Area.

Foram incluídos na amostra apenas artigos em inglês, espanhol e português que se referiam à ocorrência de dengue, independentemente da metodologia utilizada, e que estivessem disponíveis para acesso completo, gratuito e que tenham sido publicados entre 1945 e 2017. Foram excluídas cartas ao editor e revisões, além daqueles cujo tema abordado não correspondia ao objetivo desta pesquisa.

Inicialmente, procedeu-se à busca na base de dados com a utilização dos descritores e a seleção do período da publicação. Em seguida, foi realizada a leitura do título e dos resumos dos artigos encontrados, sendo excluídos aqueles que não estavam relacionados ao objetivo e à pergunta de investigação. Posteriormente, os artigos componentes da amostra final foram lidos na íntegra para análise.

A associação dos descritores na base do Web of Science identificou 60 referências. Ao selecionar apenas os artigos, a amostra foi reduzida para 56 publicações. Após a leitura dos títulos e dos resumos, 21 artigos foram excluídos por não ter dengue como objeto de estudo e ter, como foco, aspectos entomológicos. Por fim, 35 artigos foram lidos na íntegra e compuseram a amostra da revisão integrativa de literatura, para posterior separação em categorias.

\section{RESULTADOS}

Entre os 35 artigos científicos, foram encontradas as publicações de 2004 a 2017, embora o último ano tenha apresentado maior número, com cinco artigos, seguido de 2008 e 2016 com quatro em cada ano. Os anos de 2009 e 2015 tiveram três publicações cada um, enquanto 2007, 2010, 2013 e 2014 participaram com dois artigos cada. Os demais tiveram um artigo em cada ano. Com relação ao idioma, 29 foram escritos em inglês, quatro em português e dois em espanhol. O Brasil foi espaço geográfico de análise em 14 artigos, seguido de Argentina, Tailândia e Taiwan, com três em cada. Colômbia, Estados Unidos e Vietnã foram espaço de duas publicações cada um. Austrália, China, Índia, Itália, México e Trinidad e Tobago foram os países dos seis artigos restantes. 


\section{O Quadro 1 sintetiza os objetivos e os resultados de cada publicação componente da amostra.}

Quadro 1. País de estudo, objetivo e principais resultados dos artigos localizados na base de dados Web of Science (1945-2017), que contribuem para o entendimento da dinâmica da transmissão da dengue.

\begin{tabular}{|lcl}
\hline \multicolumn{1}{|c}{ Título } & País & \multicolumn{1}{c}{ Objetivo } \\
\hline $\begin{array}{l}\text { Ascertaining the impact of public rapid } \\
\text { transit system on spread of dengue in } \\
\text { urban settings }{ }^{12}\end{array}$ & Taiwan & $\begin{array}{l}\text { Investigar o papel do sistema de metrô, } \\
\text { com mais de } 50 \text { milhões de passageiros/ } \\
\text { ano como um fator que contribui para a } \\
\text { difusão da dengue em áreas ao redor do } \\
\text { sistema nos últimos anos. }\end{array}$ \\
$\begin{array}{l}\text { Modelo bayesiano para el estudio Colômbial } \\
\text { de la enfermedad del dengue en el } \\
\text { departamento de Atlántico, Colombia, } \\
\text { años } 2010 \text { a } 2013^{13}\end{array}$ & $\begin{array}{l}\text { Estudar a relação entre os casos de } \\
\text { dengue e variáveis sociais, geográficas } \\
\text { e econômicas de 23 municípios da } \\
\text { Colômbia, mediante o uso de modelos } \\
\text { espaciais bayesianos para o período } \\
2010 \text { a 2013. }\end{array}$
\end{tabular}

Individual and interactive effects of socioecological factors on dengue fever at fine spatial scale: a geographical detectorbased analysis ${ }^{14}$
China Examinar os impactos individuais e interativos dos fatores sócio ecológicos sobre a dengue.
Principais resultados

A difusão da dengue é um fenômeno complexo que envolve muitos fatores. A mobilidade urbana, de metrô, é apenas um deles, e o índice usado para relacionar mobilidade e surtos não agrega todos os fatores envolvidos. No entanto, é útil e pode ser considerado entre outros índices para o estudo das epidemias.

As variáveis sociais e o crescimento desordenado dos centros urbanos foram os fatores que mais influenciaram no aumento do número de casos de dengue.

O risco de infecção por dengue apresentou associação positiva com densidade rodoviária, temperatura do ambiente, nível de urbanização, centros urbanos e precipitação, e associação negativa com renda per capita, cobertura de vegetação e represas.

Analysis of spatial mobility in subjects from a Dengue endemic urban locality in Morelos State, Mexico ${ }^{15}$

México Estudar a micro e a macromobilidade de sujeitos infectados por dengue em um município endêmico.

O estudo sugere que casos adultos têm pape importante na dispersão da dengue, uma vez que mostraram se movimentar mais do que contatos intradomiciliares e controles fora do domicílio, facilitando assim, a infecção dos mosquitos em espaços fora do domicílio.

Distribución espacial del mosquito Aedes aegypti (Diptera: Culicidae) en el área rural de dos municipios de Cundinamarca, Colombia $^{16}$

Spatial Variations in Dengue Transmission Tailândia in Schools in Thailand ${ }^{17}$ of Aedes aegypti Breeding Sites, in the Context of a Dengue Control Program in Tartagal (Salta Province, Argentina) ${ }^{18}$

Spatial pattern evolution of Aedes aegypti breeding sites in an Argentinean city without a dengue vector control programme $^{19}$

Incorporating the human-Aedes mosquito interactions into measuring the spatial risk of urban dengue fever the spatial risk of urban dengue fever ${ }^{20}$
Colômbia Determinar a probabilidade da presença do vetor Ae. aegypti na área rural dos municípios de de Anapoima e La Mesa, Cundinamarca, Colômbia.
A região com maior probabilidade de presença do vetor foi próxima aos centros urbanos. No entanto, o índice de Breteau foi de $34 \mathrm{em}$ Anapoima e 51 em La Mesa, indicando que existe risco de transmissão na área rural dos municípios.

Os resultados sugerem que as infecções de dengue foram localizadas na escola e nas salas de aula. A escola tinha um grande número e diferentes tipos de locais para reprodução dos vetores. partir do conhecimento de infecções por dengue e abundância de vetores nesses locais.

Argentina Analisar a dinâmica espaço temporal dos locais de reprodução do Ae.aegypti e o efeito das ações de controle na população por 5 anos, em Tartagal.

Argentina Conhecer e analisar a dinâmica espaçotemporal dos locais de reprodução do Ae. aegypti em Clorinda, com relação à paisagem, usando a entropia máxima, a fim de gerar um modelo de nicho de vetores.

Taiwan Esclarecer os efeitos espaciais dos lugares com aglomeração humana para acessar o risco de exposição à dengue. consequentemente, promove acúmulo de água
Os hotspots de mosquitos estavam concentrados nas periferias, regiões com déficit de água potável, especialmente durante o verão, o que, em um conjunto de recipientes no peridomicílio.

O modelo obtido mostrou relação entre a distribuição de locais para reprodução e disponibilidade de água, aglomeração em centros urbanos e cobertura de terrenos.

O risco de infecção por DENV esteve, negativamente, correlacionado com a distância de escolas, templos e cinemas. Indivíduos próximos a esses locais tiveram risco maior de serem picados por Ae. aegypti. Os locais de atividades sociais com existência frequente do vetor devem ser incorporados nas áreas de risco para dengue e planejamento de intervenções. 


\begin{tabular}{|c|c|c|}
\hline Título & País & Objetivo \\
\hline $\begin{array}{l}\text { Spatial Distribution of Dengue in a Brazilian } \\
\text { Urban Slum Setting: Role of Socioeconomic } \\
\text { Gradient in Disease Risk }\end{array}$ & Brasil & $\begin{array}{l}\text { Examinar se características específicas } \\
\text { de comunidades urbanas estão } \\
\text { associadas com o risco de dengue. }\end{array}$ \\
\hline $\begin{array}{l}\text { High Resolution Spatial Analysis of Habitat } \\
\text { Preference of Aedes albopictus (Diptera: } \\
\text { Culicidae) in an Urban Environment }{ }^{22}\end{array}$ & Itália & $\begin{array}{l}\text { Conhecer os habitats preferidos do Ae. } \\
\text { albopictus em áreas urbanas, a fim de } \\
\text { auxiliar na prevenção e no controle das } \\
\text { doenças transmitidas por esse vetor. }\end{array}$ \\
\hline $\begin{array}{l}\text { The Spread of Dengue in an Endemic } \\
\text { Urban Milieu-The Case of Delhi, India }{ }^{23}\end{array}$ & Índia & $\begin{array}{l}\text { Descrever a epidemiologia espaço- } \\
\text { temporal da dengue, identificando } \\
\text { fatores de risco socioeconômicos. }\end{array}$ \\
\hline
\end{tabular}

Exploratory space-time analysis of dengue incidence in Trinidad: a retrospective study using travel hubs as dispersal points, 1998-2004 ${ }^{24}$
Trinidade Compreender a dinâmica espaçoTobago temporal da dengue para trazer evidências para os serviços de saúde que possam reduzir o impacto da doença.
Near real-time characterisation of urban environments: a holistic approach for monitoring dengue fever risk areas ${ }^{25}$

Water, sanitation and health: An intraurban comparison in the municipality of Caraguatatuba, Brazil ${ }^{26}$
Tailândia

Prover informação sobre a incidência de dengue com relação à cobertura de terra e estruturas urbanas.
Brasil Entender a possível inter-relação entre a distribuição espacial do saneamento básico e a epidemia de dengue de 2013.

Principais resultados

Regiões com baixas condições socioeconômicas estiveram associadas com o risco aumentado de dengue. Vegetação, radiação solar e mês de captura (agosto, setembro e outubro - verão europeu) mostraram significância na explicação do acúmulo de ovos do mosquito.

O risco de dengue diminui à medida que se distancia da floresta. Há uma alta heterogeneidade na incidência de dengue em áreas com a mesma condição socioeconômica, com grande variação ao longo do ano. Áreas pobres com alta densidade populacional foram afetadas, mas áreas de melhores condições socioeconômicas também foram infectadas, provavelmente devido à sua localização central, onde ocorre muita mobilidade.

O estudo evidenciou que as maiores incidências ocorrem em áreas onde já existe história de dengue em outros anos. Foi identificada a formação de clusters próximo a estações de transporte, com predominância de movimento do meio rural para o urbano.

Os casos estiveram concentrados em áreas com grande densidade populacional, cercada de densa vegetação. Analisando a proximidade, também foi observado que a maioria dos casos estavam próximos às instituições, igrejas e aos mercados.

A provisão e a qualidade dos serviços de fornecimento de água é um elemento chave para compreender a epidemia de dengue no município. Entretanto, é importante considerar que a dengue é um fenômeno de múltiplas causas, e políticas públicas devem ser voltadas para todos esses aspectos (população, vetor e dinâmica do vírus), para que sejam efetivas no controle da doença.

Geospatial analysis: a study about dengue ${ }^{27} \quad$ Brasil Descrever e analisar o espaço geográfico dos coeficientes de incidência de dengue segundo área urbana da Região Norte do município de Palmas/TO, Brasil.

Os resultados mostraram que surtos não estiveram presentes apenas em áreas residenciais, e as áreas de maior risco foram aquelas verdes, comerciais e pouco habitadas.

Population Movement and Vector-Borne Disease Transmission: Differentiating

Taiwan Comparar os padrões epidemiológicos, de difusão e possíveis determinantes entre casos viajantes e não viajantes para esclarecer a participação do deslocamento humano na transmissão da dengue. Cases $^{28}$

A análise dos clusters espaço-temporais mostrou que os casos não associados com viajantes se agruparam dentro de $100 \mathrm{~m}$, em uma semana, enquanto os casos relacionados a viajantes se difundiram de 2 a $4 \mathrm{~km}$ e entre 1 e cinco semanas. As viagens se apresentaram como fator de risco que contribuíram para a difusão da epidemia. Entre não viajantes, principalmente idosos e donas de casa, há risco de formação de pequenos clusters, enquanto entre os viajantes, o risco se dissipa para áreas geográficas maiores, em epidemias de grande escala.

Co-occurrence Patterns of the Dengue Vector Aedes aegypti and Aedes mediovitattus, a Dengue Competent

Estados Desenvolver um modelo preditivo Unidos de áreas onde as espécies de Aedes se sobrepõem, para conduzir coleta de amostras de Ae. mediovittatus e identificar infecção pelo DENV, focar intervenções e reduzir as futuras epidemias de dengue.
A presença do Ae. aegypti mostrou relação com áreas de maior densidade urbana e de árvores, altas temperaturas e posterior período de chuvas, provavelmente quando ocorreu acúmulo de água para sua reprodução. 


\begin{tabular}{|lcl|}
\hline \multicolumn{1}{|c}{ Título } & País & \multicolumn{1}{c}{ Objetivo } \\
\hline $\begin{array}{l}\text { Quantifying the Emergence of Dengue in } \\
\text { Hanoi,Vietnam: } 1998-2009^{30}\end{array}$ & Vietnã & $\begin{array}{l}\text { Descrever o padrão temporal da } \\
\text { incidência de dengue e sua associação } \\
\text { com as variáveis climáticas locais. }\end{array}$
\end{tabular}

Population Density, Water Supply, and the Risk of Dengue Fever in Vietnam: Cohort Study and Spatial Analysis ${ }^{31}$

\section{Vietnã Analisar a interação entre densidade} populacional e falta de água encanada como causa de surtos de dengue e identificar áreas de maior risco para infecção.

Socio-geographical factors in vulnerability to dengue in Thai villages: a spatial regression analysis ${ }^{32}$

Quantifying the Spatial Dimension of Austrália Dengue Virus Epidemic Spread within a Tropical Urban Environment ${ }^{33}$

Seroprevalence and risk factors for dengue infection in socioeconomically distinct areas of Recife, Brazil ${ }^{34}$

Spatial analysis of dengue and the socioeconomic context of the city of Rio de Janeiro (Southeastern Brazil) ${ }^{35}$

The spatial distribution of Aedes aegypti and Aedes albopictus in a transition zone, Rio de Janeiro, Brazil ${ }^{36}$
Tailândia Analisar preditores sociogeográficos para ocorrência de dengue em uma província semi-urbana.

Analisar o padrão de difusão do DENV-2 e quantificar a relação entre a difusão da dengue e localização do caso índice da epidemia.

Brasil Estimar a prevalência de dengue entre áreas privilegiadas e não privilegiadas e identificar fatores de risco individuais e de área para infecção em três áreas urbanas.

Brasil Analisar a epidemia de dengue em relação ao contexto socioeconômico, de acordo com áreas geográficas.
Brasil Avaliar a variação sazonal e espacial da abundância de larvas de Ae. aegypti e Ae. albopictus por meio de uma
Principais resultados

O padrão identificado revelou o caráter cíclico da doença. O clima influenciou em mudanças na abundância e sobrevivência do vetor, na frequência de picadas e no tempo que o mosquito leva para se tornar infectado e produzir doença em humanos. Períodos de fortes ventos estiveram associados com a redução nas notificações de dengue.

O risco de dengue foi maior em áreas rurais do que em urbanas, o que foi explicado pela ausência de água encanada.

Regiões mais próximas de áreas urbanas e famílias com menores rendas tiveram maior vulnerabilidade à dengue.

Os clusters foram identificados com distância máxima de $800 \mathrm{~m}$ da residência do caso índice. A movimentação humana e o número de suscetíveis colaboraram para a difusão da epidemia.

A prevalência de infecção foi inversamente proporcional às condições socioeconômicas. Ter idade avançada, viver em uma casa e ter maior número de habitantes/cômodo foram fatores de risco para a positividade no inquérito.

As variáveis que mostraram correlação significativa foram: percentagem de casas conectadas com a rede pública de água, casas com máquinas de lavar e densidade populacional em área urbana. Problemas relacionados ao saneamento básico contribuem para o aumento do risco de dengue. pequena escala espacial de zona de transição entre uma área urbana e uma área florestal do Rio de Janeiro.

Análise espacial da ocorrência de dengue

Brasil Analisar a ocorrência de dengue e sua relação com as condições de vida em Nova Iguaçu.

Os padrões espaciais indicaram que áreas com desigualdades sociais e próximas a vias de acesso estavam mais suscetíveis à doença.

Iguaçu, Estado do Rio de Janeiro, Brasil ${ }^{37}$

Spatial point analysis based on dengue surveys at household level in central

Brasil Identificar as áreas de risco espacial para infecção, a partir dos resultados de inquéritos de soroprevalência para dengue.

O Ae. aegypti foi mais abundante em áreas urbanas(comunidades), com picos nas estações chuvosas.

A infecção por dengue teve maior prevalência entre adultos e idosos e entre aqueles com níveis de escolaridade menores. Foi encontrada heterogeneidade espacial para áreas de risco da doença.

Brasil Descrever os padrões espacial e O resultado evidenciou maior concentração de temporal das epidemias de dengue em Belo Horizonte, Minas Gerais, Brasil, entre 1996 e 2002, analisando o endereço de residência como marcador do local de exposição.

Indicadores sociodemográficos e a Brasil Analisar a distribuição espacial da epidemia de dengue em 2002, no Estado do Rio de Janeiro, Brasil ${ }^{40}$ epidemia de dengue no Estado do Rio de Janeiro em 2002 e suas relações com as variáveis sociodemográficas. casos no grupo de idosos (mulheres) e crianças, que, supostamente, permanecem mais em casa no período diurno. A transmissão viral é contínua ao longo do ano, com picos sazonais.

Os resultados mostraram associação entre as maiores incidências de dengue e localidades caracterizadas pela crescente urbanização e por déficit na rede de canalização e abastecimento de água

Brasil Avaliar a existência de correlação espacial da incidência de dengue e identificar variáveis demográficas, socioeconômicas e ambientais que expliquem a dependência espacial.
Os resultados mostraram dependência espacial e associação da incidência alta com casas térreas, falta de coleta de lixo e esgoto, baixa renda, analfabetismo, maior número de residentes por domicílio, maior $\%$ de mulheres chefes de família. 


\begin{tabular}{|c|c|c|c|}
\hline Título & País & Objetivo & Principais resultados \\
\hline $\begin{array}{l}\text { Habitat segregation of dengue vectors } \\
\text { along an urban environmental gradient } \mathrm{t}^{42}\end{array}$ & $\begin{array}{l}\text { Estados } \\
\text { Unidos }\end{array}$ & $\begin{array}{l}\text { Investigar as diferenças de habitat } \\
\text { terrestre de Ae. aegypti e Ae. } \\
\text { mediovittatus em San Juan, Porto Rico. }\end{array}$ & $\begin{array}{l}\text { A presença de Ae. aegypti foi maior em áreas } \\
\text { urbanas com alta densidade populacional, } \\
\text { indicando que esses são locais com potencial de } \\
\text { infecção dos mosquitos pela picada de humanos } \\
\text { doentes. }\end{array}$ \\
\hline $\begin{array}{l}\text { Spatial Vulnerability to Dengue in a } \\
\text { Brazilian Urban Area During a 7-Year } \\
\text { Surveillance }^{43}\end{array}$ & Brasil & $\begin{array}{l}\text { Avaliar a associação entre variáveis } \\
\text { socioeconômicas, demográficas e de } \\
\text { infraestrutura urbana, com as áreas } \\
\text { de risco para ocorrência de dengue e } \\
\text { persistência de transmissão. }\end{array}$ & $\begin{array}{l}\text { Os fatores que melhor caracterizaram as áreas } \\
\text { de risco foram baixa escolaridade, baixa renda, } \\
\text { densidade familiar, proporção de crianças e } \\
\text { idosas. } \\
\text { É importante considerar os diferentes níveis de } \\
\text { exposição da população para explicar o padrão } \\
\text { heterogêneo de distribuição da dengue em } \\
\text { ambientes urbanos. }\end{array}$ \\
\hline $\begin{array}{l}\text { Spatial distribution pattern of oviposition } \\
\text { in the mosquito Aedes aegypti in relation } \\
\text { to urbanization in Buenos Aires: southern } \\
\text { fringe bionomics of an introduced vector }{ }^{44}\end{array}$ & Argentina & $\begin{array}{l}\text { Analisar o padrão espacial de oviposição } \\
\text { do Ae. aegypti em Buenos Aires City e } \\
\text { sua relação com variáveis demográficas } \\
\text { e ambientais. }\end{array}$ & $\begin{array}{l}\text { O padrão espacial de oviposição do Ae. aegypti } \\
\text { parece estar relacionado à urbanização. } \\
\text { Os ambientes urbanos devem ter maior } \\
\text { disponibilidade de fontes para criadouro. }\end{array}$ \\
\hline $\begin{array}{l}\text { Household survey of dengue infection in } \\
\text { Central Brazil: spatial point pattern analysis } \\
\text { and risk factor assessment }{ }^{46}\end{array}$ & Brasil & $\begin{array}{l}\text { Conhecer a soroprevalência de infecção } \\
\text { por dengue e fatores de risco individuais } \\
\text { e coletivos. }\end{array}$ & $\begin{array}{l}\text { O risco de infecção esteve associado às maiores } \\
\text { idades, à pouca escolaridade e à baixa renda. }\end{array}$ \\
\hline
\end{tabular}

\section{DISCUSSÃO}

Os artigos encontrados referiam-se à distribuição espacial da dengue, relacionando a incidência da doença com fatores socioambientais, e puderam ser divididos em sete assuntos principais: mobilidade urbana, densidade populacional, fatores sociodemográficos, disponibilidade de água, temperatura, vegetação e urbanização. A separação desses artigos nessas categorias é relevante, pois evidencia os aspectos mais comumente abordados nas produções sobre dengue que fazem uso das ferramentas da análise espacial. Essas informações nos permitem reconhecer o estado da arte a respeito dos elementos envolvidos na dinâmica de transmissão da dengue, realçando sua participação nesse processo apontando, assim, os fatores dignos de atenção na abordagem da vigilância epidemiológica.

\section{Mobilidade urbana}

A mobilidade urbana por via terrestre ou aérea, mostrou-se como um dos aspectos relevantes no processo de difusão da dengue $^{12,14}$. O estudo de Wen et al. (2012), mostrou que os clusters formados ao redor dos casos de pessoas que viajam chegam a ser vinte vezes maiores, quando comparados aos nãoviajantes ${ }^{28}$. A movimentação, especialmente de adultos que desenvolvem mais atividades de trabalho, em turnos diurnos e em locais distantes da residência - colabora para a dispersão do vírus para espaços além do domicílio ${ }^{15}$. Ainda, Telle et al. (2016) pontuam a participação dos residentes de áreas de periferia, mais vulneráveis e mais afetadas, que se deslocam para áreas centrais e com melhor condição socioeconômica, em que a circulação de pessoas é intensa ${ }^{23}$. Gil et al. (2016) também enfatizam que a mobilidade humana facilita a transmissão dos vírus e proliferação em outras localidades, desde que haja presença do vetor ${ }^{47}$. Para fins de vigilância, no que tange à mobilidade urbana, Vazquez-Prokopec et al. (2010) recomendam medidas de mitigação, entre elas, ações de controle específicas em regiões usualmente descobertas pelos serviços de saúde e incorporação de ferramentas dos sistemas de informação de geoprocessamento para visualização dos raios dos clusters, a fim de orientar os limites para as ações de contenção ${ }^{33}$.

\section{Densidade populacional}

Com relação à densidade populacional, alguns estudos evidenciaram o aumento na ocorrência da dengue e presença do vetor em ambientes com maior aglomeração de pessoas, como escolas, salas de aula, cinemas, mercados, igrejas e seus arredores ${ }^{17,20,26,30}$. Sabe-se que a presença do Ae. aegypti em áreas de maior densidade urbana - mesmo que não seja em grande densidade vetorial - mantém o ciclo de transmissão ativo em que há doentes e disponibilidade de suscetíveis ${ }^{48}$. A interação de pessoas no mesmo ambiente facilita a ocorrência da infecção do vetor a partir do humano infectado e posterior picada em outros humanos suscetíveis ${ }^{42}$. Além disso, em ambientes domésticos, o maior número de residentes/domicílio também é um fator de risco importante para a infecção, considerando a distância percorrida no voo do mosquito ${ }^{34,41,43}$. Estudos realizados no Brasil apontam que a aglomeração urbana - especialmente nas favelas - pode influenciar a incidência de dengue, pois se manifesta aliada a outros fatores característicos das desigualdades sociais, também propícios à proliferação do vetor $^{35,45}$. 


\section{Fatores sociodemográficos}

Além da vida nas favelas, entre os fatores sociodemográficos identificados nas publicações e relacionados à transmissão da dengue em meios urbanos, destacaram-se: baixa renda, viver em casas térreas, baixa escolaridade, população com idade avançada e crianças.

Estudos referem que áreas pobres, com alta densidade populacional são mais afetadas pela dengue ${ }^{18,23}$. Kikuti et al. (2015) evidenciaram que, em região de absoluta pobreza, o maior risco de infecção por dengue justifica-se pela disponibilidade de criadouros para o Ae. aegypti ${ }^{21}$. Nessas localidades, é comum a ausência de coleta de lixo e de água contínua, devido aos obstáculos geográficos e à infraestrutura precária, o que resulta em acúmulo de lixo e depósitos de água em recipientes para uso doméstico, criando locais propícios à reprodução do vetor ${ }^{49}$.

Pessoas que vivem em casas térreas também foram consideradas sob risco, quando comparadas àquelas que moram em apartamentos ${ }^{34,41}$. Estudos prévios mostraram que a oviposição do mosquito é menor em construções mais altas, o que torna a participação dos prédios pequena nos índices de infestação ${ }^{44,50}$.

As publicações de Siqueira Junior et al. (2008) mostraram que a incidência de dengue foi inversamente proporcional à escolaridade ${ }^{38,46}$, resultado também revelado por Almeida (2007), Mondini (2008) e Gonzáles et al. (2017) ${ }^{13,41,43}$. Embora saibamos que o analfabetismo não tenha relação direta com a infecção, é importante discutir que famílias em que o chefe do domicílio possui pouca escolaridade acabam por possuir baixa renda e, assim, vivem em locais de maior aglomeração, onde se apresentam situações de vulnerabilidade favoráveis à manutenção do ciclo infeccioso $32,37,41,43,45,46$.

Outros estudos encontraram associação entre dengue e a presença de crianças e idosos ${ }^{28,34,38,39}$. A participação desses grupos favorece a manutenção de pequenos clusters próximos ao domicílio, pois são pessoas que permanecem boa parte do tempo em casa, enquanto os adultos jovens e trabalhadores participam mais ativamente na dispersão da doença ${ }^{28}$.

\section{Disponibilidade de água}

A disponibilidade de água foi citada em estudos abordando as chuvas e os períodos sazonais, bem como naqueles que se referiam ao déficit de água potável e deficiências no sistema de distribuição. Os estudos de Teixeira et al. (2008) e Almeida et al. (2009) no Brasil e de Schmidt et al. (2011) no Vietnã, mostraram relação inversa entre incidência de dengue e o percentual de população com água canalizada. Com a falta de abastecimento, a água é armazenada precariamente em depósitos nos peridomicílios, criadouros potenciais para oviposição do vetor $^{31,35,40}$. A esse respeito, Johansen et al. (2013) enfatizam que a provisão e a qualidade dos serviços de fornecimento de água são fatores importantes para compreender as epidemias de dengue ${ }^{26}$.

Em um inquérito entomológico realizado na Argentina, Espinosa et al. (2016) revelaram que, entre os fatores ambientais, a deficiência na distribuição de água é responsável por $48 \%$ e $12 \%$ da concentração de vetores em municípios sem e com programas de controle do vetor, respectivamente ${ }^{18,19}$. Outros estudos encontrados na literatura demonstram que é possível predizer espacialmente a distribuição da dengue a partir da relação da reprodução do vetor com a distribuição de água e chuva no espaço ${ }^{18,51}$. Foi demonstrado que em espaços geográficos em que ocorreram períodos chuvosos, a população de vetores aumentou ${ }^{51}$, com posterior aparecimento de casos e ocorrência de surtos ${ }^{52}$. Honório et al. (2009) e Little et al. (2011) evidenciaram picos na frequência de mosquitos após esses períodos ${ }^{29,36}$, enquanto outros autores destacam o caráter cíclico da doença, explicitando a relação entre as chuvas, sazonalidade e epidemias ${ }^{30,39}$.

\section{Temperatura}

Informações sobre temperatura também se destacaram entre os fatores climáticos ligados à proliferação do vetor. Coung et al. (2011) e Little (2011) encontraram associação entre o aumento da temperatura média e a abundância e sobrevivência do vetor no Vietnã e em Porto Rico, respectivamente ${ }^{29,30}$. Na Itália, evidências estatísticas confirmaram que a radiação solar é significativa para explicar a abundância de ovos encontrados, pois eleva a temperatura nos locais de oviposição, especialmente nos meses de agosto e setembro, que correspondem ao verão europeu $^{22}$. No estudo realizado na China, também foi encontrada associação entre a infecção por dengue e temperaturas mais altas, destacando-se que, quando a combinação de chuva e temperatura são inseridas em um modelo interativo de análise, o impacto na incidência de dengue chega a ser 12 vezes maior do que outros fatores, como densidade populacional e renda, por exemplo. Além disso, é interessante lembrar que, em altas temperaturas, as pessoas passam mais tempo fora dos domicílios e com janelas abertas, facilitando, assim, a picada e a entrada do vetor no ambiente doméstico ${ }^{14}$. Por fim, Almeida (2008) enfatiza que a temperatura do ambiente é um dos três fatores responsáveis pela redução do número de casos, junto do controle vetorial e do esgotamento de suscetíveis ${ }^{39}$.

\section{Vegetação}

Sobre a vegetação, as publicações mostraram que há maior predisposição para encontrar o Ae. aegypti e seus ovos em áreas com árvores, provavelmente porque sua sombra oferece um habitat favorável22,29. Tun-Lin et al (2000), enfatizam que o material orgânico, aquele entre folhagens ou abaixo das árvores tende a produzir Ae. aegypti adultos com desenvolvimento mais rápido e melhor sobrevivência das larvas ${ }^{53}$. Com relação à doença, estudos mostraram que muitos clusters de áreas residenciais eram circulados por áreas verdes ${ }^{25,27}$. Ainda, vale lembrar que, em residências em que não há presença de árvores, as plantas ornamentais, vasos e pratos de plantas têm papel importante como criadouros para o mosquito ${ }^{54,55}$. 


\section{Urbanização}

Estudos sobre a disposição do vetor em diferentes ambientes mostraram que a população de Ae. aegypti é maior em centros urbanas, quando comparada ao Ae. albopictus, que é mais encontrado em as áreas rurais ${ }^{36}$. Em uma pesquisa realizada apenas em áreas rurais da Colômbia, Cabezas et al. (2017) encontraram que a presença de vetores foi maior naqueles sítios mais próximos das cidades, tendo sido encontradas larvas viáveis em tanques para armazenamento de água para consumo, pois nesse país $41 \%$ dessas áreas não contam com abastecimento de água por aquedutos ${ }^{16}$. Na Argentina, a maior densidade vetorial também esteve associada à urbanização e à densidade populacional, o que pode ser explicado tanto pela disponibilidade de criadouros, como de sangue, para repasto do vetor ${ }^{19,44}$.

Junto da densidade populacional, a urbanização foi um fator muito discutido nos artigos, pois o crescimento desordenado e acelerado da população em ambientes urbanos propicia condições ecológicas favoráveis à transmissão da dengue ${ }^{13,32}$; isso porque, geralmente, a aglomeração humana em pequenos domicílios vem acompanhada de má qualidade habitacional, em construções feitas sem planejamento, sem esgotamento sanitário, com distribuição insuficiente de água e em regiões sem coleta de lixo ${ }^{14,40}$.

Em suma, percebe-se que é difícil isolar os fatores relacionados à dinâmica de transmissão da dengue, uma vez que todos parecem de certa forma imbricados nas relações sociais da população, perpassando suas atividades econômicas, de trabalho, mobilidade, o crescimento desordenado das áreas urbanas, a situação de vida e de moradia e o acesso ao saneamento básico. Todas essas condições, associadas ao clima tropical, à presença do vetor e aos fatores intrínsecos (de imunidade populacional e esgotamento de suscetíveis), facilitam a propagação do vírus e sua dispersão entre a população ${ }^{52}$.

Nesse sentido, estudos de análise espacial merecem destaque por sua capacidade de identificar áreas críticas, regiões geográficas de maior risco para a ocorrência da doença, buscando relações possivelmente explicativas. A utilização dos SIG, aliada a técnicas estatísticas especializadas, permite obtenção de resultados confiáveis que possam servir de guia para os serviços de vigilância epidemiológica e ambiental. 0 desafio posto ainda é criar interfaces amigáveis entre usuários e esses sistemas ${ }^{6,39}$.

Buscando melhores resultados para além das técnicas estatísticas e informações georreferenciadas, a interpretação da análise espacial exige a percepção das relações entre espaço e sociedade, determinadas por condições econômicas e sociais, para melhor elaborar diagnósticos e ações em saúde ${ }^{56}$.

Neste estudo, a revisão integrativa mostrou a pluralidade de fatores envolvidos na ocorrência da dengue, fato que reitera a necessidade de considerar a inter e a transdisciplinaridade na análise da distribuição espacial dessa arbovirose, integrando os conhecimentos biológicos, sociais e geográficos 57 .

\section{CONCLUSÕES}

Nas Américas, a dengue tem-se destacado pelo rápido poder de disseminação e capacidade de produzir surtos e, no Brasil, é uma doença de caráter endêmico, com alta magnitude, vulnerabilidade e transcendência, na medida em que acarreta absenteísmo das atividades diárias e, em casos graves, anos potenciais de vida perdidos.

Nesse contexto, a análise espacial se mostra como uma ferramenta da informática em saúde muito útil para o estudo das dinâmicas de difusão espacial e temporal que permite observar áreas com maior risco epidemiológico, tanto pela localização de focos com potencial de proliferação do vetor, bem como os fatores sociais e demográficos, que inevitavelmente predizem a ocorrência de doença. Os SIG possibilitam o conhecimento de áreas e períodos com maior risco, a fim de produzir alertas para as vigilâncias epidemiológica e ambiental, tanto para o controle do vetor, quanto na organização da assistência necessária ao atendimento dos casos.

No entanto, com base nos artigos encontrados na revisão, foi possível verificar que a produção está concentrada principalmente entre os pesquisadores das arboviroses. Ainda é um desafio que a incorporação das ferramentas de análise espacial seja maior no cotidiano dos serviços de saúde pública, servindo como instrumento para auxiliar a tomada de decisão e ação oportuna de enfrentamento para a redução da incidência de casos.

\section{REFERÊNCIAS}

1. Lima Neto AS, Cavalcanti LPG, Araujo WN, Rouquayrol MZ. Epidemiologia Descritiva: Características e Possibilidades de Uso. In: Rouquayrol MZ, Gurgel M, organizadores. Epidemiologia e Saúde. 7. ed Rio de Janeiro: MedBook; 2013. p. 65-96.

2. Czeresnia D, Ribeiro AM. O conceito de espaço em epidemiologia: uma interpretação histórica e epistemológica. Cad Saude Publica. 2000 Set;16(3):595605. doi: http://dx.doi.org/10.1590/S0102-311X2000000300002.

3. Barata RCB, Werneck GL. Observação e Registro de Fenômenos Epidemiológicos (Tempo, Espaço, Indivíduos e Populações). In: Almeida Filho $\mathrm{N}$, Barreto ML, organizadores. Epidemiologia e Saúde: Fundamentos, Métodos, Aplicações. Rio de Janeiro: Guuanabara Koogan; 2011. p. 127-49.
4. Barreto F, Teixeira MG, Barreto ML, Barcellos C. Difusão espacial de doenças transmissíveis: uma importante perspectiva de análise epidemiológica a ser resgatada. In: Barcellos C, organizador. A geografia e o contexto dos problemas de saúde. Rio de Janeiro: Abrasco; 2008. p. 384

5. Breilh J. Epidemiologia crítica: ciênmcia emancipadora e interculturalidade. Rio de Janeiro: Fiocruz; 2006. 317 p.

6. Carvalho MS, Souza-Santos R. Análise de dados espaciais em saúde pública: métodos, problemas, perspectivas. Cad Saude Publica [Internet]. 2005 [acesso 2018 Jun 27]; 21(2):361-78. Disponível em: http://www.scielo.br/scielo. php?script=sci_arttext $\&$ pid=S0102-311X2005000200003\&lng=pt\&nrm=iso\&tl $\mathrm{ng}=\mathrm{pt}$ 
7. Peiter PC, Barcellos CC, Rojas LBI, Gondim GMM. Espaço Geográfico e Epidemiologia. In: Ministério da Saúde [BR]. Secretaria de Vigilância em Saúde. Fundação Osvaldo Cruz. Santos SM, Barcellos C, organizadores. Abordagens Espaciais na Saúde Pública. Brasília: Ministério da Saúde; 2006. p. 136.

8. Teixeira MG, Costa MCN, Paixão ES, Carmo EH, Barreto FR, Penna GO Conquistas do SUS no enfrentamento das doenças transmissíveis. Cien Saude Colet [Internet]. 2018 Jun [acesso 2018 Jun 27]; 23(6):1819-28. Disponível em: $\quad$ http://www.scielo.br/scielo.php?script=sci_arttext\&pid=S1413$81232018000601819 \& \operatorname{lng}=p t \& n r m=i s o \& t \operatorname{lng}=p t$

9. Souza MT, Silva MD, Carvalho R. Revisão integrativa: o que é e como fazer. Einstein. 2010; 8(1 Pt 1):102-6.

10. Soares $C B$, Hoga LAK, Peduzzi $M$, Sangaleti $C$, Yonekura T, Silva DRAD. Revisão integrativa: conceitos e métodos utilizados na enfermagem. Rev Esc Enferm USP. 2014 Abr;48(2):329-39. doi: http://dx.doi.org/10.1590/S00806234201400002000020 .

11. Mendes KD, Silveira RC, Galvão CM. Revisão Integrativa: método de pesquisa para a incorporação de evidências na saúde e na enfermagem. Texto context - enferm. 2008;17(4):758-64. doi: http://dx.doi.org/10.1590/S010407072008000400018

12. Sanna M, Hsieh YH. Ascertaining the impact of public rapid transit system on spread of dengue in urban settings. Sci Total Environ [Internet]. 2017 Nov [acesso 2018 Jun 27];598:1151-9. Disponível em: https://www.ncbi.nlm.nih.gov/ pubmed/28499330 . doi: http://dx.doi.org/10.1016/j.scitotenv.2017.04.050

13. González AAM, Ortiz Beltrán FG, Santa Guzmán LF. Modelo bayesiano para el estudio de la enfermedad del dengue en el departamento de Atlántico, Colombia, años 2010 a 2013. Perspect Geográfica [Internet]. 2017 [acesso 2018 Jun 27]; 22(2). Disponível em: http://revistas.uptc.edu.co/revistas/index.php/ perspectiva/article/view/7603

14. Cao Z, Liu T, Li X, Wang J, Lin H, Chen L, et al. Individual and interactive effects of socio-ecological factors on dengue fever at fine spatial scale: A geographical detector-based analysis. Int J Environ Res Public Health. 2017;14(7). doi: 10.3390/ijerph14070795.

15. Falcón-Lezama JA, Santos-Luna R, Román-Pérez $S$, Martínez-Vega RA, Herrera-Valdez MA, Kuri-Morales ÁF, et al. Analysis of spatial mobility in subjects from a Dengue endemic urban locality in Morelos State, Mexico. PLoS One. 2017 Fev; 12(2):e0172313. doi: 10.1371/journal.pone.0172313.

16. Cabezas L, Cabanzo W, Santa F, Olano VA, Sarmiento D, Vargas S, et al. Distribución espacial del mosquito Aedes aegypti (Diptera: Culicidae) en el área rural de dos municipios de Cundinamarca, Colombia. Biomédica. 2017;37:24. doi:10.7705/biomedica.v34i2.3469

17. Ratanawong $P$, Kittayapong $P$, Olanratmanee $P$, Wilder-Smith $A$, Byass $P$, Tozan $Y$, et al. Spatial variations in dengue transmission in schools in Thailand. PLoS One. 2016 Sep;11(9):1-16. doi: 10.1371/journal.pone.0161895

18. Espinosa M, Weinberg D, Rotela CH, Polop F, Abril M, Scavuzzo CM. Temporal Dynamics and Spatial Patterns of Aedes aegypti Breeding Sites, in the Context of a Dengue Control Program in Tartagal (Salta Province, Argentina). PLoS Negl Trop Dis. 2016 May;10(5):1-21. doi: 10.1371/journal.pntd.0004621.

19. Espinosa MO, Polop F, Rotela CH, Abril M, Scavuzzo CM, Sano FM, et al. Spatial pattern evolution of Aedes aegypti breeding sites in an Argentinean city without a dengue vector control programme. Geospat Health. 2016 Nov; 11(471):307-17. doi: 10.4081/gh.2016.471.

20. Wen $\mathrm{TH}$, Lin $\mathrm{MH}$, Teng HJ, Chang NT. Incorporating the human-Aedes mosquito interactions into measuring the spatial risk of urban dengue fever. Appl Geogr [Internet]. 2015 Aug [cited 2018 Jun 27]; 62:256-66. Available at: https://www.sciencedirect.com/science/article/pii/S014362281500123X\#! doi: http://dx.doi.org/10.1016/j.apgeog.2015.05.003

21. Kikuti M, Cunha GM, Paploski IAD, Kasper AM, Silva MMO, Tavares AS, et al. Spatial distribution of dengue in a Brazilian Urban slum setting: Role of socioeconomic gradient in disease risk. PLoS Negl Trop Dis. 2015 Jul; 9(7):1-18. doi: 10.1371/journal.pntd.0003937.

22. Cianci D, Hartemink N, Zeimes CB, Vanwambeke SO, lenco A, Caputo B. High resolution spatial analysis of habitat preference of Aedes albopictus (Diptera: Culicidae) in an urban environment. J Med Entomol. 2015 May; 52(3):329-35. doi: 10.1093/jme/tjv026

23. Telle O, Vaguet A, Yadav NK, Lefebvre B, Cebeillac A, Nagpal BN, et al. The Spread of Dengue in an Endemic Urban Milieu--The Case of Delhi, India. PLoS One. 2016 Jan;11(1):e0146539. doi: 10.1371/journal.pone.0146539.

24. Sharma KD, Mahabir RS, Curtin KM, Sutherland JM, Agard JB, Chadee DD. Exploratory space-time analysis of dengue incidence in Trinidad: A retrospective study using travel hubs as dispersal points, 1998-2004. Parasites and Vectors. 2014 Jul; 7(1):1-11. doi: 10.1186/1756-3305-7-341.

25. Sarfraz MS, Tripathi NK, Kitamoto A. Near real-time characterisation of urban environments: a holistic approach for monitoring dengue fever risk areas. Int J Digit Earth. 2014;7(11):916-34. doi: https://doi.org/10.1080/17538947.20 13.786144

26. Johansen IC, Carmo RL, Bueno MCD. Water, sanitation and health: An intraurban comparison in the municipality of Caraguatatuba, Brazil. Water Int. 2013 Nov;38(7):888-901. doi: http://dx.doi.org/10.1080/02508060.2013.856834

27. Cavalcante MPR, Oliveira C, Simão FB, Lima PR, Monteiro PS. Geospatial analysis: a study about dengue. ACTA Paul Enferm. 2013;26(4):360-8. doi: http://dx.doi.org/10.1590/\$0103-21002013000400010

28. Wen T, Lin M, Fang C. Disease V, Cases ND. Population Movement and Vector-Borne Disease Transmission: Differentiating Spatial - Temporal Diffusion Patterns of Commuting and Noncommuting Dengue Cases. Ann Assoc Am Geogr. 2012;102(5):1026-37.

29. Little E, Barrera R, Seto KC, Diuk-Wasser M. Co-occurrence patterns of the dengue vector Aedes aegypti and aedes mediovitattus, a dengue competent mosquito in Puerto Rico. Ecohealth. 2011 Sep;8(3):365-75. doi: 10.1007/ s10393-011-0708-8

30. Cuong HQ, Hien NT, Duong TN, Phong TV, Cam NN, Farrar J, et al. Quantifying the emergence of dengue in Hanoi, Vietnam: 1998-2009. PLoS Negl Trop Dis. 2011Sep; 5(9):1-7. doi: 10.1371/journal.pntd.0001322.

31. Schmidt W-P, Suzuki M, Thiem VD, White RG, Tsuzuki A, Yoshida L-M, et al. Population Density, Water Supply, and the Risk of Dengue Fever in Vietnam: Cohort Study and Spatial Analysis. PLoS Med [Internet]. 2011 Aug [acesso 2018 Jun 27]; 8(8):e1001082. doi: http://dx.plos.org/10.1371/journal.pmed.1001082

32. Tipayamongkholgu $M$, Lisakulruk $S$. Socio-geographical factors in vulnerability to dengue in thai villages: A spatial regression analysis. Geospat Health. 2011 May; 5(2):191-8. doi: 10.4081/gh2011.171.

33. Vazquez-Prokopec GM, Kitron U, Montgomery B, Horne P, Ritchie SA Quantifying the spatial dimension of dengue virus epidemic spread within a tropical urban environment. PLoS Negl Trop Dis. 2010 Dec; 4(12):1-14. doi: 10.1371/journal.pntd.0000920.

34. Braga C, Luna CF, Martelli CMT, Souza WV, Cordeiro MT, Alexander N, et al. Seroprevalence and risk factors for dengue infection in socioeconomically distinct areas of Recife, Brazil. Acta Trop. 2010 Mar;113(3). doi: 10.1016/j. actatropica.2009.10.021

35. Almeida AS, Medronho RA, Valencia LIO. Spatial analysis of dengue and the socioeconomic context of the city of Rio de Janeiro (Southeastern Brazil). Rev Saude Publica. 2009 Aug; 43(4):666-73. doi: http://www.ncbi.nlm.nih.gov/ pubmed/19649472

36. Honório NA, Castro MG, Barros FSM, Magalhães MAFM, Sabroza PC. The spatial distribution of Aedes aegypti and Aedes albopictus in a transition zone, Rio de Janeiro, Brazil. Cad Saude Publica. 2009 Jun; 25(6):1203-14. doi: http:// 
7. Peiter PC, Barcellos CC, Rojas LBI, Gondim GMM. Espaço Geográfico e Epidemiologia. In: Ministério da Saúde [BR]. Secretaria de Vigilância em Saúde. Fundação Osvaldo Cruz. Santos SM, Barcellos C, organizadores. Abordagens Espaciais na Saúde Pública. Brasília: Ministério da Saúde; 2006. p. 136.

8. Teixeira MG, Costa MCN, Paixão ES, Carmo EH, Barreto FR, Penna GO. Conquistas do SUS no enfrentamento das doenças transmissíveis. Cien Saude Colet [Internet]. 2018 Jun [acesso 2018 Jun 27]; 23(6):1819-28. Disponível em: $\quad$ http://www.scielo.br/scielo.php?script=sci_arttext\&pid=S1413$81232018000601819 \& \operatorname{lng}=p t \& n r m=i s o \& t \operatorname{lng}=p t$

9. Souza MT, Silva MD, Carvalho R. Revisão integrativa: o que é e como fazer. Einstein. 2010; 8(1 Pt 1):102-6.

10. Soares CB, Hoga LAK, Peduzzi M, Sangaleti C, Yonekura T, Silva DRAD. Revisão integrativa: conceitos e métodos utilizados na enfermagem. Rev Esc Enferm USP. 2014 Abr;48(2):329-39. doi: http://dx.doi.org/10.1590/S00806234201400002000020 .

11. Mendes KD, Silveira RC, Galvão CM. Revisão Integrativa: método de pesquisa para a incorporação de evidências na saúde e na enfermagem. Texto context - enferm. 2008;17(4):758-64. doi: http://dx.doi.org/10.1590/S010407072008000400018.

12. Sanna M, Hsieh YH. Ascertaining the impact of public rapid transit system on spread of dengue in urban settings. Sci Total Environ [Internet]. 2017 Nov [acesso 2018 Jun 27];598:1151-9. Disponível em: https://www.ncbi.nlm.nih.gov/ pubmed/28499330 . doi: http://dx.doi.org/10.1016/j.scitotenv.2017.04.050

13. González AAM, Ortiz Beltrán FG, Santa Guzmán LF. Modelo bayesiano para el estudio de la enfermedad del dengue en el departamento de Atlántico, Colombia, años 2010 a 2013. Perspect Geográfica [Internet]. 2017 [acesso 2018 Jun 27]; 22(2). Disponível em: http://revistas.uptc.edu.co/revistas/index.php/ perspectiva/article/view/7603

14. Cao Z, Liu T, Li X, Wang J, Lin H, Chen L, et al. Individual and interactive effects of socio-ecological factors on dengue fever at fine spatial scale: A geographical detector-based analysis. Int J Environ Res Public Health. 2017;14(7). doi: 10.3390/ijerph14070795.

15. Falcón-Lezama JA, Santos-Luna R, Román-Pérez $S$, Martínez-Vega RA, Herrera-Valdez MA, Kuri-Morales ÁF, et al. Analysis of spatial mobility in subjects from a Dengue endemic urban locality in Morelos State, Mexico. PLoS One. 2017 Fev; 12(2):e0172313. doi: 10.1371/journal.pone.0172313.

16. Cabezas L, Cabanzo W, Santa F, Olano VA, Sarmiento D, Vargas S, et al. Distribución espacial del mosquito Aedes aegypti (Diptera: Culicidae) en el área rural de dos municipios de Cundinamarca, Colombia. Biomédica. 2017;37:24. doi:10.7705/biomedica.v34i2.3469

17. Ratanawong $\mathrm{P}$, Kittayapong $\mathrm{P}$, Olanratmanee $\mathrm{P}$, Wilder-Smith $\mathrm{A}$, Byass $\mathrm{P}$, Tozan $Y$, et al. Spatial variations in dengue transmission in schools in Thailand. PLoS One. 2016 Sep;11(9):1-16. doi: 10.1371/journal.pone.0161895

18. Espinosa M, Weinberg D, Rotela CH, Polop F, Abril M, Scavuzzo CM. Temporal Dynamics and Spatial Patterns of Aedes aegypti Breeding Sites, in the Context of a Dengue Control Program in Tartagal (Salta Province, Argentina). PLoS Negl Trop Dis. 2016 May;10(5):1-21. doi: 10.1371/journal.pntd.0004621.

19. Espinosa MO, Polop F, Rotela CH, Abril M, Scavuzzo CM, Sano FM, et al. Spatial pattern evolution of Aedes aegypti breeding sites in an Argentinean city without a dengue vector control programme. Geospat Health. 2016 Nov 11(471):307-17. doi: 10.4081/gh.2016.471.

20. Wen $\mathrm{TH}$, Lin $\mathrm{MH}$, Teng HJ, Chang NT. Incorporating the human-Aedes mosquito interactions into measuring the spatial risk of urban dengue fever. Appl Geogr [Internet]. 2015 Aug [cited 2018 Jun 27]; 62:256-66. Available at: https://www.sciencedirect.com/science/article/pii/S014362281500123X\#! doi: http://dx.doi.org/10.1016/j.apgeog.2015.05.003

21. Kikuti M, Cunha GM, Paploski IAD, Kasper AM, Silva MMO, Tavares AS, et al. Spatial distribution of dengue in a Brazilian Urban slum setting: Role of socioeconomic gradient in disease risk. PLoS Negl Trop Dis. 2015 Jul; 9(7):1-18 doi: 10.1371/journal.pntd.0003937.

22. Cianci D, Hartemink N, Zeimes CB, Vanwambeke SO, lenco A, Caputo B. High resolution spatial analysis of habitat preference of Aedes albopictus (Diptera: Culicidae) in an urban environment. J Med Entomol. 2015 May; 52(3):329-35. doi: 10.1093/jme/tjv026

23. Telle O, Vaguet A, Yadav NK, Lefebvre B, Cebeillac A, Nagpal BN, et al. The Spread of Dengue in an Endemic Urban Milieu--The Case of Delhi, India. PLoS One. 2016 Jan;11(1):e0146539. doi: 10.1371/journal.pone.0146539.

24. Sharma KD, Mahabir RS, Curtin KM, Sutherland JM, Agard JB, Chadee DD. Exploratory space-time analysis of dengue incidence in Trinidad: A retrospective study using travel hubs as dispersal points, 1998-2004. Parasites and Vectors. 2014 Jul; 7(1):1-11. doi: 10.1186/1756-3305-7-341.

25. Sarfraz MS, Tripathi NK, Kitamoto A. Near real-time characterisation of urban environments: a holistic approach for monitoring dengue fever risk areas. Int J Digit Earth. 2014;7(11):916-34. doi: https://doi.org/10.1080/17538947.20 13.786144

26. Johansen IC, Carmo RL, Bueno MCD. Water, sanitation and health: An intraurban comparison in the municipality of Caraguatatuba, Brazil. Water Int. 2013 Nov;38(7):888-901. doi: http://dx.doi.org/10.1080/02508060.2013.856834

27. Cavalcante MPR, Oliveira C, Simão FB, Lima PR, Monteiro PS. Geospatial analysis: a study about dengue. ACTA Paul Enferm. 2013;26(4):360-8. doi: http://dx.doi.org/10.1590/\$0103-21002013000400010

28. Wen T, Lin M, Fang C. Disease V, Cases ND. Population Movement and Vector-Borne Disease Transmission: Differentiating Spatial - Temporal Diffusion Patterns of Commuting and Noncommuting Dengue Cases. Ann Assoc Am Geogr. 2012;102(5):1026-37.

29. Little E, Barrera R, Seto KC, Diuk-Wasser M. Co-occurrence patterns of the dengue vector Aedes aegypti and aedes mediovitattus, a dengue competent mosquito in Puerto Rico. Ecohealth. 2011 Sep;8(3):365-75. doi: 10.1007/ s10393-011-0708-8

30. Cuong HQ, Hien NT, Duong TN, Phong TV, Cam NN, Farrar J, et al. Quantifying the emergence of dengue in Hanoi, Vietnam: 1998-2009. PLoS Negl Trop Dis. 2011Sep; 5(9):1-7. doi: 10.1371/journal.pntd.0001322.

31. Schmidt W-P, Suzuki M, Thiem VD, White RG, Tsuzuki A, Yoshida L-M, et al. Population Density, Water Supply, and the Risk of Dengue Fever in Vietnam: Cohort Study and Spatial Analysis. PLoS Med [Internet]. 2011 Aug [acesso 2018 Jun 27]; 8(8):e1001082. doi: http://dx.plos.org/10.1371/journal.pmed.1001082

32. Tipayamongkholgu $M$, Lisakulruk $S$. Socio-geographical factors in vulnerability to dengue in thai villages: A spatial regression analysis. Geospat Health. 2011 May; 5(2):191-8. doi: 10.4081/gh2011.171.

33. Vazquez-Prokopec GM, Kitron U, Montgomery B, Horne P, Ritchie SA Quantifying the spatial dimension of dengue virus epidemic spread within a tropical urban environment. PLoS Negl Trop Dis. 2010 Dec; 4(12):1-14. doi: 10.1371/journal.pntd.0000920.

34. Braga C, Luna CF, Martelli CMT, Souza WV, Cordeiro MT, Alexander N, et al. Seroprevalence and risk factors for dengue infection in socioeconomically distinct areas of Recife, Brazil. Acta Trop. 2010 Mar;113(3). doi: 10.1016/j. actatropica.2009.10.021

35. Almeida AS, Medronho RA, Valencia LIO. Spatial analysis of dengue and the socioeconomic context of the city of Rio de Janeiro (Southeastern Brazil). Rev Saude Publica. 2009 Aug; 43(4):666-73. doi: http://www.ncbi.nlm.nih.gov/ pubmed/19649472

36. Honório NA, Castro MG, Barros FSM, Magalhães MAFM, Sabroza PC. The spatial distribution of Aedes aegypti and Aedes albopictus in a transition zone, Rio de Janeiro, Brazil. Cad Saude Publica. 2009 Jun; 25(6):1203-14. doi: http:// 
dx.doi.org/10.1590/S0102-311X2009000600003.

37. Machado JP, Oliveira, Magalhães R, Souza-Santos R. Análise espacial da ocorrência de dengue e condições de vida na cidade de Nova Iguaçu, Estado do Rio de Janeiro, Brasil. Cad Saude Publica. 2009; 25(5):1025-34. doi: http:// dx.doi.org/10.1590/S0102-311X2009000500009.

38. Siqueira-Junior JB, Maciel IJ, Barcellos C, Souza WV, Carvalho MS, Nascimento $\mathrm{NE}$, et al. Spatial point analysis based on dengue surveys at household level in central Brazil. BMC Public Health. 2008 Oct; 8:1-9. doi: 10.1186/1471-2458-8361.

39. Almeida MCM, Assunção RM, Proietti FA, Caiaffa WT. Dinâmica intra-urbana das epidemias de dengue em Belo Horizonte, Minas Gerais, Brasil, 1996-2002. Cad Saúde Pública. 2008 Out; 24(10):2385-95.

40. Teixeira TRDA, Medronho RDA. Indicadores sócio-demográficos e a epidemia de dengue em 2002 no Estado do Rio de Janeiro, Brasil. Cad Saude Publica. 2008;24(9):2160-70. doi: http://dx.doi.org/10.1590/S0102$311 \times 2008000900022$

41. Mondini A, Chiaravalloti-Neto F. Spatial correlation of incidence of dengue with socioeconomic, demographic and environmental variables in a Brazilian city. Sci Total Environ. 2008 Apr; 393(2-3):241-8. doi: 10.1016/j. scitotenv.2008.01.010

42. Cox J, Grillet ME, Ramos OM, Amador M, Barrera R. Habitat Segregation of Dengue Vectors Along an Urban Environmental Gradient. Am J Trop Med Hyg [Internet]. 2007 May [acesso 2018 Jun 27];76(5):820-6. Available at: http:// www.ajtmh.org/content/76/5/820.long

43. Almeida MCM, Caiaffa WT, Assunção RM, Proietti FA. Spatial vulnerability to dengue in a Brazilian urban area during a 7-year surveillance. J Urban Heal. 2007 Jan; 84(3):334-45. doi: [10.1007/s11524-006-9154-2.

44. Carbajo A, Curto S, Schweigmann J. Spatial distribution pattern of oviposition in the mosquito Aedes aegypti in relation to urbanization in Buenos Aires: southern fringe bionomics of an introduced vector. Med Vet Entomol. 2006 Jun; (20):209-18.

45. Barcellos C, Pustai AK, Weber MA, Brito MRV. Identificação de locais com potencial de transmissão de dengue em Porto Alegre através de técnicas de geoprocessamento. Rev Soc Bras Med Trop. 2005 Maio-Jun; 38(3):246-50. doi: http://dx.doi.org/10.1590/S0037-86822005000300008.

46. Siqueira JB, Martelli CMT, Maciel IJ, Oliveira RM, Ribeiro MG, Amorim FP, et al. Household survey of dengue infection in central Brazil: spatial point pattern analysis and risk factors assessment. Am J Trop Med Hyg. 2004 Nov; 71(5):64651.

47. Gil JF, Palacios M, Krolewiecki AJ, Cortada P, Flores R, Jaime C, et al. Spatial spread of dengue in a non-endemic tropical city in northern Argentina. Acta Trop. 2016 Jun;158:24-31. doi: http://dx.doi.org/10.1016/j.actatropica.2016.02.003

48. Reiter P. Status of current Aedes aegypti control methodologies. In: Halstead SB, Gomez-Dantes H, organizadores. Dengue: a worldwide problem, a commom strategy. Mexico: Ministry of Health - Mexico; Rockefeller Foundation; 1992. p. 41-8.

49. Tauil PL. Urbanização e ecologia do dengue. Cad Saude Publica. 2001;17 (Suppl):99-102.

50. Seidahmed OME, Lu D, Chong CS, Ng LC, Eltahir EAB. Patterns of Urban Housing Shape Dengue Distribution in Singapore at Neighborhood and Country Scales. GeoHealth [Internet]. 2018 Jan [acesso 2018 Jun 27]; 54-67. doi: https:// doi.org/10.1002/2017GH000080

51. Ortiz PL, Rivero A, Linares Y, Pérez A, Vázquez JR. Spatial Models for Prediction and Early Warning of Aedes aegypti Proliferation from Data on Climate Change and Variability in Cuba. MEDICC Rev. 2015 Apr;17(2):20-8. PubMed PMID: 26027583.

52. Stewart-lbarra AM, Muñoz ÁG, Ryan SJ, Ayala EB, Borbor-Cordova MJ, Finkelstein JL, et al. Spatiotemporal clustering, climate periodicity, and socialecological risk factors for dengue during an outbreak in Machala, Ecuador, in 2010. BMC Infect Dis [Internet]. 2014;14(1):610. doi: https://doi.org/10.1186/ s12879-014-0610-4.

53. Tun-Lin W, Burkot TR, Kay BH. Effects of temperature and larval diet on development rates and survival of the dengue vector Aedes aegypti in north Queensland, Australia. Med Vet Entomol. 2000 Mar;14(1):31-7.

54. Varejão JBM, Santos CB, Rezende HR, Bevilacqua LC, Falqueto A. Criadouros de Aedes (Stegomyia) aegypti (Linnaeus, 1762) em bromélias nativas na Cidade de Vitória, ES. Rev Soc Bras Med Trop. 2005;38(3):238-40. doi: http://dx.doi. org/10.1590/S0037-86822005000300006.

55. Oliveira RMAB, Araújo FMC, Cavalcanti LPG. Aspectos entomológicos e epidemiológicos das epidemias de dengue em Fortaleza, Ceará, 20012012. Epidemiol e Serviços Saúde [Internet]. 2018 Fev [acesso 2018 Jun 27]; 27(1):e201704414. Disponível em: http://www.scielo.br/scielo.php?script=sci arttext\&pid=S2237-96222018000100309\&Ing=pt\&nrm=iso\&tlng=pt. doi: http://dx.doi.org/10.5123/s1679-49742018000100014.

56. Barcellos CC, Sabroza PC, Peiter P, Rojas LI. Organização espacial, saúde e qualidade de vida: análise espacial e uso de indicadores na avaliação de situações de saúde. Inf Epidemiológico do Sus [Internet]. 2002 [acesso 2018 Jun 27]; 11(3):129-38. Disponível em: https://www.arca.fiocruz.br/bitstream/ icict/713/2/BARCELLOS_Analise espacial e uso de indicadores_saude_2002.pdf

57. Costa MCN, Teixeira MGLC. A concepção de "espaço" na investigação epidemiológica. Cad Saude Publica. 1999;15(2):271-9. doi: http://dx.doi. org/10.1590/S0102-311X1999000200012.

\section{Como citar este artigo/How to cite this article:}

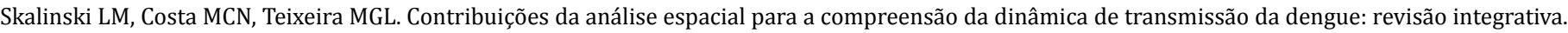
J Health Biol Sci. 2018 Jan-Mar; 7(1):53-63. 